\title{
Immunomodulator effect of ketamine on rat models of sepsis (fecal induced peritonitis): Eosinophil and monocyte modulation
}

\author{
Aswoco Andyk Asmoro, Wiwi Jaya, Asna Nasiqah, Muhammad Rodli \\ Department of Anesthesiology and Intensive Therapy, Faculty of Medicine, Brawijaya University- Dr. Saiful Anwar General \\ Hospital, Malang-Jl. Jaksa Agung Suprapto No.2, Malang, East Java, Indonesia \\ Correspondence: Aswoco Andyk Asmoro, Department of Anesthesiology and Intensive Therapy, Faculty of Medicine, Brawijaya \\ University- Dr. Saiful Anwar General Hospital, Malang Jl. Jaksa Agung Suprapto No.2, Malang, East Java, Indonesia; \\ E-mail: aandykasmoro@ub.ac.id
}

\section{Abstract}

Background \& Objective: Sepsis is a result of dysregulated inflammation and it affects the inflammatory mediator cell production. Ketamine has an immunomodulatory effect and it can be used in the sepsis immunotherapy. We conducted this study to determine the effect of ketamine on the number of eosinophils and monocytes in the rat model of sepsis.

Methodology: This research was a true animal experiment using 30 white rats (Rattus norvegicus), which were divided into six groups. Control negative group (NC) were not treated and the control positive group (PC) were the sepsis groups. Sepsis was produced by fecal induced peritonitis (FIP). Animals in the four treatment groups (A, B, C, and D) received intraperitoneal (IP) ketamine at $5 \mathrm{mg} / \mathrm{kg}$ body weight at different single hours (0,3,5 hours) and intermittently (every 2 hours), respectively, after receiving FIP. The eosinophil and monocyte counts were obtained 6 hours after FIP. The data were analyzed using One-Way ANOVA and Tukey HSD statistical test using SPSS software version $16.0(p<0.05)$.

Results: Sepsis significantly decreased the number of eosinophils but increased monocyte production. Ketamine treatment right after FIP (Group A) prevents the reduction of eosinophils in the sepsis group ( $p<0.05$ ) from 8 cells to 66 cells. Ketamine treatment in sepsis groups (Group A, B, and D) significantly reduced the monocyte production to close to normal (equivalent to NC group).

Conclusion: Sepsis significantly reduces the number of eosinophils and increases monocyte production in the rat models of sepsis. Ketamine, given soon enough after onset of sepsis helps to prevent the sepsis-induced changes in eosinophil and monocyte counts.

Key words: Eosinophil; Fecal induced peritonitis; Immunomodulation; Ketamine; Monocyte; Sepsis

Citation: Asmoro AA, Jaya W, Nasiqah A, Rodli M. Immunomodulator effect of ketamine on rat models of sepsis (fecal induced peritonitis): Eosinophil and monocyte modulation. Anaesth. pain intensive care 2020;24(3):279-283

Received: 26 August 2019; Reviewed: 22 February 2020; Accepted for 8 March 2020.

\section{Introduction}

Sepsis is a systemic dysregulated inflammatory response to infection. It is characterized by an overproduction of pro-inflammatory cytokines and has been associated with a high mortality rate. ${ }^{1,2}$ The number of patients with sepsis admitted to Intensive Care Units (ICU) increases every year. ${ }^{3}$ In Indonesia, a study shows that out of 4,774 patients admitted to the ICU, there were 504 patients diagnosed with sepsis. ${ }^{4}$ The mortality rate of sepsis is up to $70.2 \%{ }^{4}$ Medical record data collected between 2012-2013 at Dr. Saiful Anwar General Hospital, Malang found 1,026 patients diagnosed with sepsis and $788(76.8 \%)$ of them died. ${ }^{5}$

Various diagnostic markers of sepsis have been developed. C-reactive protein (CRP) and 
procalcitonin (PCT) are commonly used biomarkers. Recent research has shown that eosinophil is a specific sepsis marker and can be used as a diagnostic tool. ${ }^{6,7}$ The research showed an association between eosinophils and bacterial infections in ICU patients. An abnormal fall in the number of eosinophils (eosinopenia) can be used as a predictor of mortality in septic patients. ${ }^{8}$ Eosinopenia occurs because of the rapid sequestration of eosinophils in the peripheral circulation, suppression of eosinophil production and suppression of mature eosinophils migration. The decrease of eosinophil also happens due to the release of glucocorticoids from the adrenals stimulated by endotoxin and lipopolysaccharide. Glucocorticoids will inhibit the eosinophil release from the bone marrow, as well as their adhesion, migration, and chemotaxis. The eosinophils chemotactic inhibition trough interleukin (IL)-3, IL-5, and GM-CSF inhibition mechanism. IL-5 controls eosinophil growth, differentiation, and survival rate. Sepsis also activates the inflammatory mediator cells such as monocytes and macrophages. Overexpression of inflammatory response leads to the abnormality of the immune system and result in organ-system dysfunction. In bacterial sepsis, endotoxin and lipopolysaccharide from the bacteria will induce macrophages, dendritic cells, and neutrophils secrete pro-inflammatory cytokines such as IL-1, IL-6 and tumor necrosis factor- $\alpha(\mathrm{TNF}-\alpha){ }^{8-10}$

Ketamine is an anesthetic agent commonly used in sepsis patients. ${ }^{9}$ A study by Gurfinkel et al. ${ }^{10}$ showed that ketamine improves the survival rate of severe burn injury followed by sepsis. Ketamine also has a good effect on maintaining cardiovascular and hemodynamic stability; and it has immunomodulating activities. ${ }^{10}$ Research carried out by Shanked et al. ${ }^{11}$ showed that ketamine significantly increased the survival rates in rats with Escherichia coli-induced sepsis. ${ }^{12}$ It inhibits pro-inflammatory cytokine production through downregulation of proinflammatory gene expression pathway. ${ }^{13}$ Eosinophil and monocyte counts can be used as therapeutic targets in sepsis. This study was conducted to determine the effect of ketamine on the eosinophil and monocyte counts in rats models of sepsis.

\section{Methodology}

This animal experimental study was conducted at the clinical pathology laboratory of the Faculty of Medicine, Brawijaya University, Malang, Indonesia in December 2016-January 2017. The research was approved by the Health Research Ethics Committee of Faculty of Medicine, Brawijaya University (No.452/EC/KEPK/12/2016). This study used 30 white male rats (Rattus norvegicus), aged 5 months, weight 200-250 grams, showed active movements, and their hair did not fall out. The samples were divided into six treatment groups. The mice in negative control (NC group) were not treated. Positive control (PC group) was the sepsis group induced with fecal induced peritonitis (FIP). FIP was produced by dissolved feces from rat with normal saline until the concentration became $200 \mathrm{mg} / \mathrm{ml}$. Feces $1 \mathrm{mg} / \mathrm{g}$ were administrated intraperitoneally (IP) to induce peritonitis. The mice received ketamine $5 \mathrm{mg} / \mathrm{kg}$ body weight intraperitoneally (IP) right after FIP (0 hours) in Group A, 3 h after FIP in Group B, 5 hours after FIP in Group $\mathrm{C}$ and intermittently every 2 hours (0, 2 and 4 hours after FIP) in Group D. Peripheral blood mononuclear cells were isolated from the heart at $6 \mathrm{~h}$ after FIP. The number of eosinophils and monocyte was calculated using a hematology analyzer.

\subsection{Statistical analysis}

The data (the number of eosinophils and monocytes) were analyzed statistically using SPSS software (version 16.0, IBM Statistic, United States). The data were analyzed using the homogeneity test and normality test with $\mathrm{p}>0.05$. The data were then analyzed using the One-Way ANOVA test followed by the Tukey HSD test with $\alpha=0.05$. The significant difference happens when $\mathrm{p}<0.05$.

\section{Results}

This research showed varied results. The number of eosinophils in the normal group (NC) was 56.4 cells. The administration of FIP in the sepsis group (PC group) significantly decreases the number of eosinophils to 8.8 cells $(\mathrm{p}=0.016)$. The administration of ketamine significantly increased the number of eosinophils in group A (66.2 cells) ( $\mathrm{p}=$ $0.008)$ compare to sepsis group. The number of 


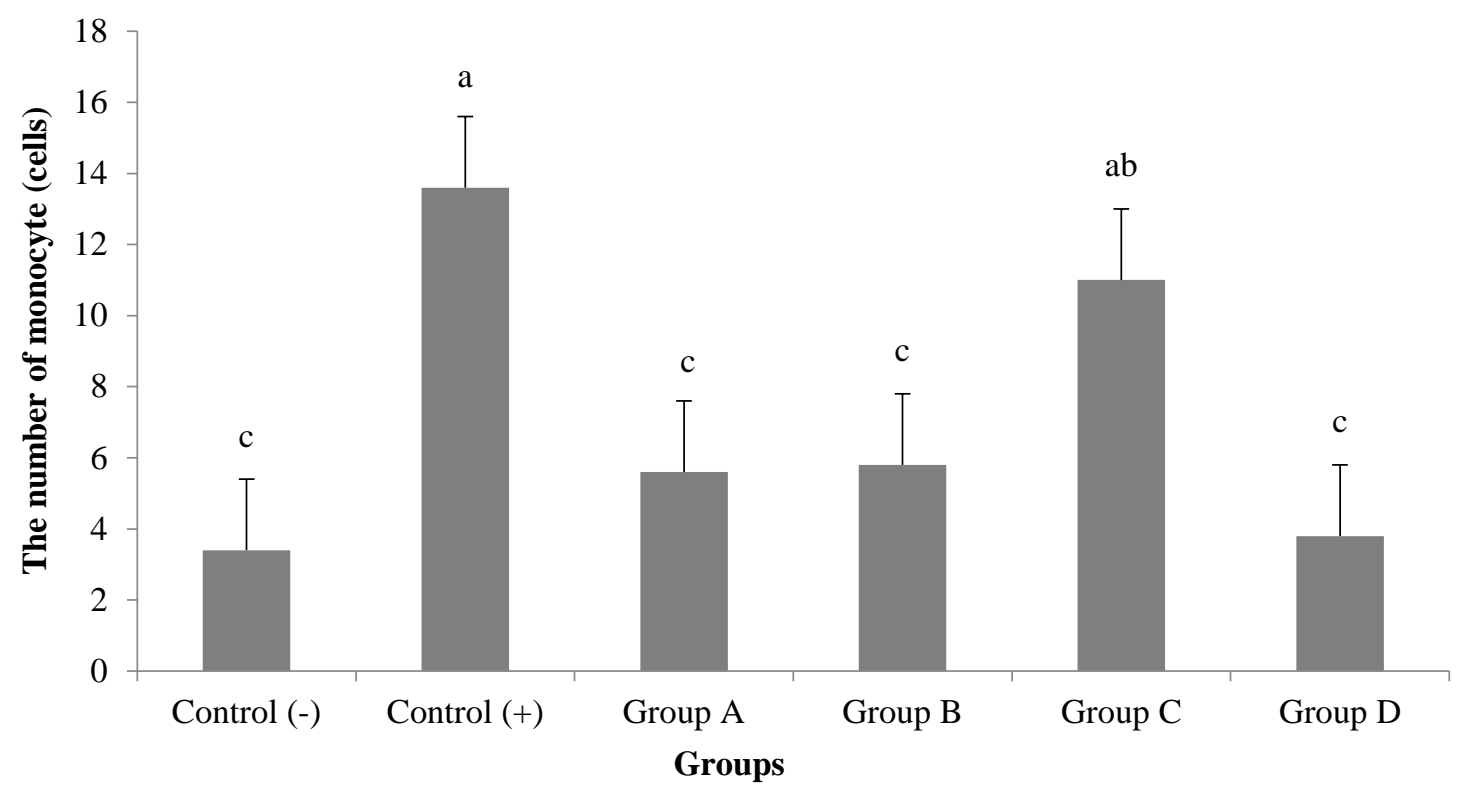

Figure 1: Number of eosinophils in each group

Group control (-): normal; Group control (+): sepsis; Group A: administration of ketamine 0 h after FIP; Group B; administration of ketamine $3 \mathrm{~h}$ after FIP, Group C: administration of ketamine $5 \mathrm{~h}$ after FIP; Group D: administration of ketamine intermittently every $2 h$. The letters above the error bar indicate when there is no statistically significant difference between the groups. Hence, the groups in this study that have a statistically significant difference are those labelled with an "ab" compared to those labelled with a "c."

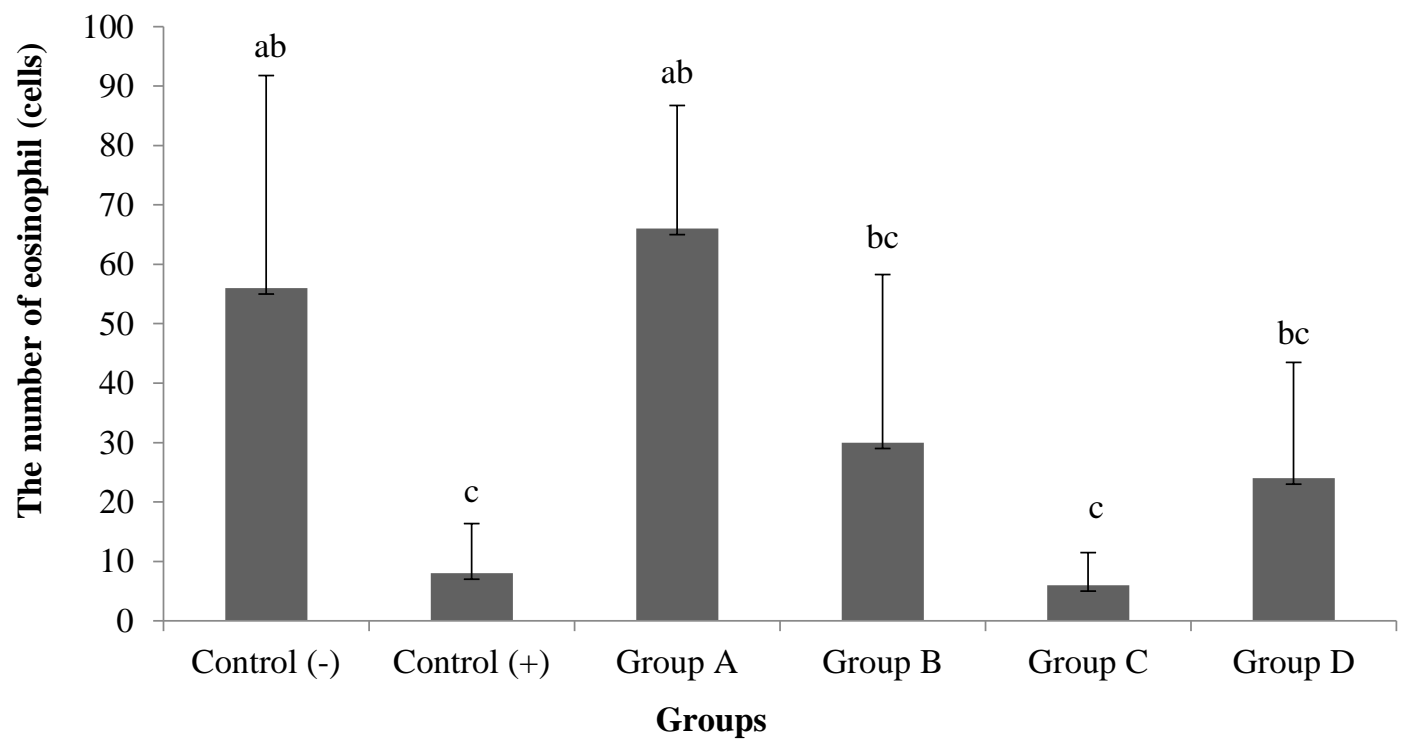

Figure 2: The number of monocyte in each group

Group control (-): normal; Group control (+): sepsis; Group A: administration of ketamine 0 h after FIP; Group B; administration of ketamine $3 \mathrm{~h}$ after FIP, Group C: administration of ketamine 5 h after FIP; Group D: administration of ketamine intermittently every $2 h$. The letters above the error bar indicate when there is no statistically significant difference between the groups. Hence, groups are statistically significantly different when their labels share no letters 
eosinophils in group B was 30.3 cells, while in group D it was 24.2 cells. Both groups were not statistically different from the sepsis group, but did show an increase in the number of eosinophils. Group $\mathrm{C}$ had fewer eosinophils than the PC group (sepsis group) (Figure 1).

The results showed a significant increase in the number of monocytes in the PC group (sepsis model) compare to normal group. The number of monocytesin the $\mathrm{NC}$ (normal) group was 3.4 cells. FIP induction significantly increased the number of monocytes in the PC group to 13.6 cells $(\mathrm{p}=0.000)$. The administration of ketamine in the sepsis group significantly caused a decrease in the number monocytes in groups A, B, and D compare to sepsis group ( $<$ 0.05). The number of monocytes in group $A$ and B was 5.6 and 5.8 cells, while in the group D was 3.8 cells $(p>0.05)$. The $p>0.05$ indicated that at the number of monocyte in groups $\mathrm{A}, \mathrm{B}$, and $\mathrm{D}$ were statistically similar to the normal group. The number of monocyte in Group C was 11 cells and it did not differ from the sepsis group significantly (Figure 2).

\section{Discussion}

Giving FIP in the PC group significantly decreased the number of eosinophils $(\mathrm{p}=0.016)$. The decrease in the eosinophil count is called eosinopenia. The result supports the study by Abidi et al. ${ }^{8}$ which states that sepsis significantly decreases the number of eosinophils. The eosinophil number in the rats, that were immediately given ketamine after FIP, were higher than the sepsis group. Ketamine prevents the decrease of eosinophil count in the rat model of sepsis and keeps it close to the normal condition. Ketamine administration in the rat that were given ketamine $3 \mathrm{~h}$ after FIP, $5 \mathrm{~h}$ after FIP and intermittently every $2 \mathrm{~h}$ did not have a significant difference from the sepsis group $(p=0.841)$. Giving ketamine in the rat that were given ketamine $5 \mathrm{~h}$ after FIP did not prevent the decrease of eosinophil count because the drop in the number of eosinophils at 5 hours after FIP had occurred by the time the ketamine had been given.

The results showed that there was a significant increase in the number of monocytes in the PC group (sepsis group) from 3.4 cells to 13.6 cells ( $\mathrm{p}=$ 0.000 ). The results of this study are similar to the research conducted by Lee and Kim. ${ }^{14}$ An increase in the number of monocytes occurs as an innate immune response..$^{15}$ The administration of ketamine in the rats that were immediately given ketamine after FIP (5.6 cells), $3 \mathrm{~h}$ after FIP (5.8 cells), and intermittently every $2 \mathrm{~h}$ ( 3.8 cells) significantly caused a decrease in the number of monocytes close to the normal group ( $\mathrm{p}>$ 0.05). Ketamine can inhibit monocyte differentiation through N-methyl-D-aspartatedependent mechanisms. ${ }^{16}$

The study showed that ketamine $5 \mathrm{mg} / \mathrm{kg}$ body weight (IP) can potentially serve as sepsis immunotherapy. Ketamine $5 \mathrm{mg} / \mathrm{kg}$ body weight can modulate both eosinophil and monocyte production. Ketamine was able to modulate the number of cells to stay in the homeostasis condition. The administration time shows a significant effect. The administration of ketamine right after FIP gives the best result in normalizing the number of eosinophils and monocytes to that of the normal condition. There was, however, no significant difference between one time administration and intermittent repeated administration of ketamine.

\section{Conclusion}

Sepsis significantly reduces the number of eosinophils and increases monocyte production in the rat models of sepsis; and ketamine, given soon enough, can help prevent the sepsis-induced changes in eosinophil and monocyte counts.

\section{Acknowledgment}

The authors acknowledge and thank the staff of Clinical Pathology Laboratory, Faculty of Medicine, Brawijaya University, Malang, Indonesia for their full support in conduction of this research.

\section{Authors' contribution}

AAN, WWJ: Conceived and designed the experiments; conducted the study; analyzed and interpreted the data

AN, ODI: Conducted the study; analyze and interpreted the data; manuscript preparation and editing 


\section{Conflict of interest}

The authors declare no conflict of interest.

\section{References}

1. Schulte W, Bernhagen J, Bucala R. cytokines in sepsis: potent immunoregulators and potential therapeutic targets-an updated view. Mediators Inflamm. 2013;2013:1- 165974. [PubMed] DOI: 10.1155/2013/165974

2. Ulloa L, Cai B, Deitch EA. Novel insights for systemic inflammation in sepsis and hemorrhage. Mediators Inflamm. 2010;2010:642462. [PubMed] DOI: 10.1155/2010/642462

3. Tannehill D. Treating Severe sepsis \& septic shock in 2012. J Blood Disord Transfus. 2013;01(S4):2-7. DOI: 10.4172/2155-9864.s4-002

4. Pradipta IS, Sodik DC, Lestari K, Parwati I, Halimah E, Diantini A, et al. Antibiotic resistance in sepsis patients: Evaluation and recommendation of antibiotic use. $\mathrm{N}$ Am J Med Sci. 2013;5(6):344-352. [PubMed] DOI: 10.4103/1947-2714.114165

5. Asmoro AA, Rakhmatullah R, Puspitasari S, Tarimah $\mathrm{K}$, Saleh SC, Widodo MA. et al. The effect of ketamine on the lipopolysaccharide-induced inflammation in in vitro culture of HUVEC. Asian Pacific J Trop Dis. 2015;5(11):894-896. DOI: 10.1016/S22221808(15)60952-5

6. Lavoignet $\mathrm{CE}$, Le Borgne $\mathrm{P}$, Slimani $\mathrm{H}$, Forato M, Kam $C$, Kauffmann $P$, et al. Relevance of eosinopenia as marker of sepsis in the emergency department. Rev Med Interne. 2016:37(11):730-734. [PubMed] DOI: 10.1016/j.revmed.2016.02.018

7. Davido B, Makhloufi S, Matt M, Calin R, Senard O, Perronne $\mathrm{C}$, et al. Changes in eosinophil count during bacterial infection: revisiting an old marker to assess the efficacy of antimicrobial therapy. Int $\mathrm{J}$ Infect Dis. 2017;61:62-66. [PubMed] DOI: 10.1016/i.ijid.2017.06.005

8. Abidi K, Khoudri I, Belayachi J, Madani N, Zekraoui A, Zeggwagh $\mathrm{AA}$, et al. Eosinopenia is a reliable marker of sepsis on admission to medical intensive care units. Crit Care. 2008;12(2):1-10. [PubMed] DOI: 10.1186/cc6883

9. Pinsky MR. Dysregulation of the immune response in severe sepsis. 2004;328(4):220-229. [PubMed] DOI: 10.1097/00000441-200410000-00005

10. Shaaban H, Daniel S, Sison R, Slim J, Perez G.
Eosinopenia: is it a good marker of sepsis in comparison to procalcitonin and C-reactive protein levels for patients admitted to a critical care unit in an urban hospital? J Crit Care. 2010;25(4):570-575. [PubMed] DOI: 10.1016/j.jcrc.2010.03.002

11. Morris C, Perris A, Klein J, Mahoney P. Anaesthesia in haemodynamically compromised emergency patients: Does ketamine represent the best choice of induction agent? Anaesthesia. 2009;64(5):532-539. [PubMed] DOI: 10.1111/j.1365-2044.2008.05835.x

12. Gurfinkel $R$, Czeiger $D$, Douvdevani A, Shapira $Y$, Artru $A A$, Sufaro $Y$, et al. Ketamine improves survival in burn injury followed by sepsis in rats. Anesth Analg. 2006;103(2):396-402. [PubMed] DOI: 10.1213/01.ane.0000226140.84281.3e

13. Shaked G, Czeiger D, Dukhno O, Levy I, Artru AA, Shapira $Y$, et al. Ketamine improves survival and suppresses IL-6 and TNFalpha production in a model of Gram-negative bacterial sepsis in rats. Resuscitation. 2004;62(2):237-242. [PubMed] DOI: 10.1016/j.resuscitation.2004.02.015

14. Peters K, Unger RE, Brunner J, Kirkpatrick CJ. Molecular basis of endothelial dysfunction in sepsis. Cardiovasc Res. 2003 Oct 15;60(1):49-57. [PubMed] DOI: 10.1016/s0008-6363(03)00397-3

15. Liu FL, Chen TL, Chen RM. Mechanisms of ketamineinduced immunosuppression. Acta Anaesthesiol Taiwan. 2012;50(4):172-177. [PubMed] DOI: 10.1016/j.aat.2012.12.001

16. Lee AJ, Kim SG. Mean cell volumes of neutrophils and monocytes are promising markers of sepsis in elderly patients. Blood Res. 2013;48(3):193-197. [PubMed] DOI: $10.5045 / \mathrm{br} .2013 .48 .3 .193$

17. Santos SS, Carmo AM, Brunialti MK, Machado FR, Azevedo LC, Assunção $M$, et al. Modulation of monocytes in septic patients: preserved phagocytic activity, increased ROS and NO generation, and decreased production of inflammatory cytokines. Intensive Care Med Exp. 2016;4(1):5. [PubMed] DOI: 10.1186/s40635-016-0078-1

18. Plevin RE, Knoll M, McKay M, Arbabi S, Cuschieri J. The role of lipopolysaccharide structure in Monocyte activation and cytokine secretion. Shock. 2016;45(1):22-27. [PubMed] DOI: 10.1097/SHK.0000000000000470

19. Laudanski K, Qing M, Oszkiel H, Zawadka M, Lapko N, et al. Ketamine affects in vitro differentiation of monocyte into immature dendritic cells. Anesthesiology. 2015;(3):628-641. [PubMed] DOI: 10.1097/ALN.0000000000000783 